\title{
About the Nature of the Spectrum of the Periodic Problem for the Heat Equation with a Deviating Argument
}

\author{
I. Orazov, A. Shaldanbayev, and M. Shomanbayeva \\ M. Auezov South Kazakhstan State University, 5 Tauke Khan Avenue, Shymkent 160012, Kazakhstan \\ Correspondence should be addressed to M. Shomanbayeva; mtshomanbaeva@mail.ru \\ Received 17 May 2013; Revised 24 July 2013; Accepted 3 September 2013 \\ Academic Editor: Valery Covachev
}

Copyright (C) 2013 I. Orazov et al. This is an open access article distributed under the Creative Commons Attribution License, which permits unrestricted use, distribution, and reproduction in any medium, provided the original work is properly cited.

We study the nature of the spectrum of the periodic problem for the heat equation with a lower-order term and with a deviating argument. A significant influence of the lower-order term on the correct solvability of this problem is found. We obtain a criterion for the strong solvability of the above-mentioned problem.

\section{Introduction}

In paper [1] we constructed a spectral theory of a model differential equation of first order with a deviating argument. The main idea of the article [1] has been further developed in $[2,3]$.

The equation that we studied belongs to a class of functional-differential equations. These equations have only recently become a subject of research of individual authors; particularly, such equations are studied in [4-6]. Functionaldifferential equations have been actively studied recently by some authors in papers $[7,8]$.

In this paper using the methods of paper [1] we have found a solution of the mixed problem for the heat equation with a deviating argument. As a result we got that the classical solvability of the boundary value problem requires a certain smoothness of the right-hand side of the equation (see (11) of Theorem 4) and the strong solvability of the problem is provided by the properties of the coefficient of the lowerorder term of the equation (Theorem 11).

Let $\Omega \subset R^{2}$ be a rectangle bounded by the following segments:

$$
\begin{array}{ll}
A B: 0 \leq t \leq T, x=0, & B C: 0 \leq x \leq l, t=T, \\
C D: 0 \leq t \leq T, x=l, & D A: 0 \leq x \leq l, t=0 .
\end{array}
$$

We denote by $C^{2,1}(\Omega)$ the set of functions $u(x, t)$ that are twice continuously differentiable with respect to the variable $x$ and once continuously differentiable with respect to the variable $t$. The boundary of the area $\Omega$ is a set of segments $B=A B \cup A D \cup C D$.

Periodic Problem. Find a solution of the equation

$$
L u=u_{t}(x, T-t)+u_{x x}(x, t)+a u_{x}=f(x, t)
$$

satisfying the conditions

$$
\begin{gathered}
\left.u\right|_{t=0}=0, \\
\left.u\right|_{x=0}-\left.u\right|_{x=l}=0,\left.\quad u_{x}\right|_{x=0}-\left.u_{x}\right|_{x=l}=0,
\end{gathered}
$$

where $f \in L^{2}(\Omega)$ and $a$ is constant.

Further the coefficient $a$ will be called the coefficient of influence.

Definition 1. The function $u(x, t) \in L^{2}(\Omega)$ is called a strong solution of (2)-(4) if there exists a sequence of functions $\left\{u_{n}\right\} \in C^{2,1}(\Omega) \cap C^{1,0}(\bar{\Omega}), n=1,2,3, \ldots$, that satisfies the boundary conditions of the problem and such that $\left\{u_{n}\right\}$ and $\left\{L u_{n}\right\}, n=1,2,3, \ldots$, converge, respectively, to $u$ and $f$.

Definition 2. The boundary value problem (2)-(4) is called strongly solvable if a strong solution of the problem exists for any right-hand side $f(x, t) \in L^{2}(\Omega)$ and this solution is unique. 
The aim of this study is to investigate the nature of the spectrum and the influence of the lower-order term on the strong solvability of the problem (2)-(4) in the space $L^{2}(\Omega)$.

Using Fourier method we obtained the conditions of the existence of a strong solution of the boundary value problem (2)-(4) in the space $L^{2}(\Omega)$ in the form of Theorem 4 . With the help of the spectral theory of linear operators we have established criteria of strong solvability of this problem, presented in the form of Theorem 11, which is the main result of this paper.

\section{Results}

Problem about Spectrum. Examine the nature of the spectrum of the functional-differential operator

$$
\begin{gathered}
L u=u_{t}(x, T-t)+u_{x x}(x, t)+a u_{x}, \\
D(L)=\left\{u \in C^{2,1}(\Omega) \cap C(\bar{\Omega}),\right. \\
\left.\left.u\right|_{t=0}=\left.u\right|_{x=0}-\left.u\right|_{x=l}=\left.u_{x}\right|_{x=0}-\left.u_{x}\right|_{x=l}=0\right\} .
\end{gathered}
$$

Theorem 3. The spectral problem

$$
\begin{gathered}
L u=u_{t}(x, T-t)+u_{x x}(x, t)+a u_{x}(x, t)=\lambda u(x, t), \\
\left.u\right|_{t=0}=\left.u\right|_{x=0}-\left.u\right|_{x=l}=\left.u_{x}\right|_{x=0}-\left.u_{x}\right|_{x=l}=0
\end{gathered}
$$

has an infinite number of eigenvalues:

$$
\begin{array}{r}
\lambda_{m n}=(-1)^{n}\left(n+\frac{1}{2}\right) \frac{\pi}{T}-\left(\frac{2 m \pi}{l}\right)^{2}+\frac{2 m \pi i}{l} a, \\
m=0, \pm 1, \pm 2, \ldots, \quad n=0,1,2, \ldots,
\end{array}
$$

and the corresponding eigenfunctions

$$
\begin{array}{r}
u_{m n}(x, t)=\frac{2}{\sqrt{T l}} \exp \left(\frac{2 m \pi i}{l} x\right) \cdot \sin \left(n+\frac{1}{2}\right) \frac{\pi t}{T}, \\
m=0, \pm 1, \pm 2, \ldots, \quad n=0,1,2, \ldots,
\end{array}
$$

which form an orthonormal basis of the space $L^{2}(\Omega)$, where $\Omega=[0, l] \times[0, T]$.

Proof. By method of separation of variables we get two spectral problems. The first problem is the Sturm-Liouville problem with Dirichlet condition. The second problem is the Cauchy problem for the first-order equation with deviating arguments, which is studied in detail in [1]. The rest is elementary.

Theorem 4. For the existence and uniqueness of a strong solution of the boundary problem (2)-(4), it is necessary and sufficient to fulfill the condition

$$
\begin{aligned}
& {\left[(-1)^{n}\left(n+\frac{1}{2}\right) \cdot \frac{\pi}{T}-\left(\frac{2 m \pi}{l}\right)^{2}-\frac{2 m \pi}{l} \cdot \operatorname{Im} a\right]^{2}} \\
& +\left(\frac{2 m \pi}{l} \cdot \operatorname{Re} a\right)^{2} \neq 0, \quad \forall m=0, \pm 1, \pm 2, \ldots, n=0,1,2, \ldots
\end{aligned}
$$

When this condition is fulfilled a strong solution of the problem exists and has the form

$$
u(x, t)=\sum_{m=-\infty}^{\infty} \sum_{n=0}^{\infty} \frac{\left(f, u_{m n}\right)}{\lambda_{m n}} \cdot u_{m n}(x, t)
$$

for all $f(x, t) \in L^{2}(\Omega)$ satisfying

$$
\sum_{m=-\infty}^{\infty} \sum_{n=0}^{\infty}\left|\frac{\left(f, u_{m n}\right)}{\lambda_{m n}}\right|^{2}<+\infty,
$$

where the eigenvalues $\lambda_{m n}$ and the eigenfunctions $u_{m n}(x, t)$ are given by (7) and (8).

The proof is omitted since this theorem is a simple consequence of the preceding theorem.

Theorem 5. If $a+\bar{a}=0$, then the differential operator (5) is essentially self-adjoint in the space $H=L^{2}(\Omega)$, where $\Omega=$ $[0, l] \times[0, T]$ is a rectangle, lying in the upper half-plane $(x, t) \in$ $R^{2}$.

Proof. It easily follows from the symmetry of the operator $L$ and the completeness of its eigenvectors.

From Theorems 4 and 5 there follows the Theorem 6.

Theorem 6. If

(a) $\operatorname{Re} a=0$,

(b) $(-1)^{n}(n+1 / 2) \cdot(\pi / T)-(2 m \pi / l)^{2}-(2 m \pi / l) \cdot \operatorname{Im} a \neq 0$,

then the inverse operator $(\bar{L})^{-1}$ exists, which is also self-adjoint.

Proof. From Theorem 4 there follows the existence of the operator $(\bar{L})^{-1}$; the rest follows from the series of equalities $\left(\bar{L}^{-1}\right)^{*}=\left(\bar{L}^{*}\right)^{-1}=(\bar{L})^{-1}$, in which Theorem 5 and the known equality $\left(A^{-1}\right)^{*}=\left(A^{*}\right)^{-1}$ were used.

Definition 7. A linear operator $A$ (not necessarily bounded) in space $H$ is called normal if it is densely defined and closed and satisfies the condition $A^{*} A=A A^{*}$.

Lemma 8 (see [9]). Let $A$ be a densely defined operator in a Hilbert space $H$. Then
(a) $A^{*}$ is closed;
(b) A allows a closure if and only if $D\left(A^{*}\right)$ is dense, and in this case $\bar{A}=A^{* *}$;
(c) if A allows a closure, then $(\bar{A})^{*}=A^{*}$.

Lemma 9 (see [10]). Let A be a normal operator in the space H. Then
(a) $D(A)=D\left(A^{*}\right)$;
(b) $\|A x\|=\left\|A^{*} x\right\|$ for $\forall x \in D(A)$;
(c) $A$ is the maximum normal operator. 
Using these lemmas we obtain the next theorem.

Theorem 10. If $a+\bar{a} \neq 0$, then the closure of the operator $L$ is a normal operator; that is, the equality $\bar{L}^{*} \bar{L}=\bar{L} \bar{L}^{*}$ is satisfied.

Proof. This formula can be verified directly.

\section{About the Nature of the Spectrum of the Operator $\bar{L}$}

Theorem 11. (a) If $\operatorname{Re} a \neq 0$, then there exists an inverse operator $\bar{L}^{-1}$, which is normal and compact. We have the estimate

$$
\left\|\bar{L}^{-1}\right\| \leq K^{-1}, \quad K=\max \left\{\frac{\pi}{2 T}, \frac{2 \pi}{l}|\operatorname{Re} a|\right\} .
$$

The spectrum of the operator $\bar{L}$ is discrete, that is, has no limit points in the finite part of the plane.

(b) If $\operatorname{Re} a=0, \operatorname{Im} a \neq\left((-1)^{n}(n+1 / 2) / 2 m\right) \cdot(l / T)-$ $2 m \pi / l, n=0,1,2, \ldots, m=0, \pm 1, \pm 2, \ldots$, and both values of $2 \pi T / l^{2}$ and $T \cdot \operatorname{Im} a / l$ are rational, then the inverse operator $\bar{L}^{-1}$ exists and is bounded but not compact. The operator $\bar{L}$ is self-adjoint; its spectrum consists of an infinite number of eigenvalues, among which there are an infinite number of infinite multiple eigenvalues.

(c) If $\operatorname{Re} a=0, \operatorname{Im} a \neq\left((-1)^{n}(n+1 / 2) / 2 m\right) \cdot(l / T)-2 m \pi / l$, $n=0,1,2, \ldots, m=0, \pm 1, \pm 2, \ldots$ and at least one of the values of $2 \pi T / l^{2}$ and $T \cdot \operatorname{Im} a / l$ is irrational, then the inverse operator $\bar{L}^{-1}$ exists, but is not bounded. The operator $\bar{L}$ is self-adjoint and its spectrum consists of an infinite number of eigenvalues and continuous spectrum filling the entire real axis $(-\infty,+\infty)$. The points of the continuous spectrum are the limit points of eigenvalues.

(d) If $\operatorname{Re} a=0$ and $\operatorname{Im} a=\left(\left((-1)^{n}(n+1 / 2)\right) / 2 m\right) \cdot(l / T)-$ $2 m \pi / l$ for some values $n=0,1,2, \ldots ; m=0, \pm 1, \pm 2, \ldots$, the inverse operator $\bar{L}^{-1}$ does not exist. The operator $\bar{L}$ is selfadjoint. If both values of $2 \pi T / l^{2}$ and $T \cdot \operatorname{Im} a / l$ are rational, the spectrum consists of an infinite number of eigenvalues, among which there are an infinite number of infinite multiple eigenvalues. If at least one of the values of $2 \pi T / l^{2}, T \cdot \operatorname{Im} a / l$ is irrational, then the spectrum of the operator $\bar{L}$ fills the entire real line $(-\infty,+\infty)$.

Proof. In the work [11] Weyl introduced the concept of a sequence uniformly distributed modulo 1 and also proved a criterion of uniform distribution. In the same paper he gave examples of sequences distributed uniformly modulo 1 . The simplest of these sequences is the sequence $1 \xi, 2 \xi, 3 \xi$,... with some irrational number $\xi$.

Weyl's First Theorem. If $\varphi(z)$ is a polynomial with a constant term $\alpha_{0}$ and not all coefficients of $\varphi(z)-\alpha_{0}$ are rational, then the sequence of numbers $\varphi(1), \varphi(2), \varphi(3), \ldots$ is distributed uniformly dense everywhere.

In particular:

Weyl's Second Theorem. If $\xi$ is some irrational number, the sequence of points $1 \xi, 4 \xi, 9 \xi, 16 \xi, 25 \xi, \ldots$ when winding of a real axis around a circle of length 1 covers it evenly dense everywhere. The same will hold if the squares of numbers are replaced by their cubes or fourth degrees, and so forth.

Next, we show that the set of eigenvalues $\left\{\lambda_{m n}\right\}$ is compacted with an increase in the indices $n$ and $m$.

The eigenvalues that we found are of the form

$$
\begin{aligned}
& \lambda_{m n}=(-1)^{n}\left(n+\frac{1}{2}\right) \frac{\pi}{T}-\left(\frac{2 m \pi}{l}\right)^{2}+\frac{2 m \pi i}{l} a, \\
& a \neq 0, \quad m=0, \pm 1, \pm 2, \ldots, \quad n=0,1,2, \ldots
\end{aligned}
$$

Let us consider the neighborhood of the origin. If it was a limit point of the set of eigenvalues, then the inverse operator $(\bar{L})^{-1}$ would be unbounded.

If $\lambda_{m n} \rightarrow 0$ for some subsequence, then

$$
\begin{aligned}
\left|\lambda_{m n}\right|^{2}= & \left((-1)^{n}\left(n+\frac{1}{2}\right) \frac{\pi}{T}-\left(\frac{2 m \pi}{l}\right)^{2}-\frac{2 m \pi}{l} \cdot \operatorname{Im} a\right)^{2} \\
& +\left(\frac{2 m \pi}{l} \cdot \operatorname{Re} a\right)^{2} \longrightarrow 0
\end{aligned}
$$

This is impossible when $\operatorname{Re} a \neq 0$. If $\operatorname{Re} a \neq 0$, then

$$
\begin{aligned}
&\left|\lambda_{m n}\right|^{2} \geq\left(\frac{2 \pi}{l} \operatorname{Re} a\right)^{2}, \quad \forall m=1,2, \ldots, \\
& \Longrightarrow\left|\lambda_{m n}\right| \geq\left(\frac{2 \pi}{l} \operatorname{Re} a\right), \quad \forall m=1,2, \ldots, \\
&\left|\lambda_{0 n}\right|^{2}=\left[\left(n+\frac{1}{2}\right) \frac{\pi}{T}\right]^{2} \geq\left(\frac{\pi}{2 T}\right)^{2}, \\
&\left|\lambda_{0 n}\right| \geq \frac{\pi}{2 T}, \quad \forall n=0,1,2, \ldots .
\end{aligned}
$$

Hence,

$$
\begin{array}{r}
\left|\lambda_{m n}\right| \geq \max \left\{\frac{\pi}{2 T}, \frac{2 \pi}{l}|\operatorname{Re} a|\right\}, \quad \forall m=0, \pm 1, \pm 2, \ldots ; \\
n=0,1,2, \ldots
\end{array}
$$

Thus, when Re $a \neq 0$ the inverse operator $\bar{L}^{-1}$ exists and is bounded. If a subsequence $\left\{\lambda_{k l}\right\}$ of the sequence $\left\{\lambda_{m n}\right\}$ converges to a point $\lambda_{0}$ in the complex plane, then the sequence $\left\{\left|\lambda_{k l}\right|\right\}$ is bounded; therefore, the second index $l$ takes only a finite number of values. Then the first index takes a finite number of values too. We have a contradiction, since assumption on $\left\{\lambda_{k l}\right\}$ is an infinite convergent sequence. Therefore, the sequence $\left\{\lambda_{m n}\right\}$ has no limit points in the finite part of the complex plane $\lambda$, which means that the spectrum of the operator $\bar{L}$ is discrete.

Now we will investigate whether the operator $\bar{L}^{-1}$ is compact. Any subsequence of the sequence $\left\{\lambda_{m n}\right\}, m=$ $0, \pm 1, \pm 2, \ldots, n=0,1,2 \ldots$, tends to infinity. In fact, let $\left\{\lambda_{i j}\right\}$ be an arbitrary infinite subsequence of the sequence $\left\{\lambda_{m n}\right\}$. Then two situations are possible: 
(1) either the first index takes an infinite number of values, then

$$
\left|\lambda_{i j}\right|^{2} \geq\left(\frac{2 i \pi}{l} \operatorname{Re} a\right)^{2}, \Longrightarrow\left|\lambda_{i j}\right| \longrightarrow+\infty,
$$

(2) or the first index takes a finite number of values, while the second index takes an infinite number of values; therefore

$$
\begin{aligned}
\left|\lambda_{i j}\right|^{2}= & {\left[(-1)^{j}\left(j+\frac{1}{2}\right) \frac{\pi}{T}-\left(\frac{2 i \pi}{l}\right)^{2}\right.} \\
& \left.-\frac{2 i \pi}{l} \cdot \operatorname{Im} a\right]^{2}+\left(\frac{2 i \pi}{l} \cdot \operatorname{Re} a\right)^{2} \\
\geq & {\left[\left(j+\frac{1}{2}\right) \frac{\pi}{T}+(-1)^{j+1}\left(\frac{2 i \pi}{l}\right)^{2}\right.} \\
& \left.+(-1)^{j+1} \frac{2 i \pi}{l} \cdot \operatorname{Im} a\right]^{2} \rightarrow \infty .
\end{aligned}
$$

Due to the fact that $j \rightarrow+\infty$, the second and third terms are the bounded quantities.

Lemma 12 (see [12]). For the complete continuity of the operator of the normal type it is necessary and sufficient to fulfill the condition

$$
\lim _{n \rightarrow+\infty} \lambda_{n}=0
$$

On the basis of Lemma 12 and (17) and (18) it follows that the inverse operator $\bar{L}^{-1}$ is completely continuous. Therefore its spectrum is discrete.

Now consider the case $\operatorname{Re} a=0$.

In this case, the eigenvalues have the form

$$
\begin{array}{r}
\lambda_{m n}=(-1)^{n}\left(n+\frac{1}{2}\right) \frac{\pi}{T}-\left(\frac{2 m \pi}{l}\right)^{2}-\frac{2 m \pi}{l} \operatorname{Im} a \\
\operatorname{Im} a \neq 0 .
\end{array}
$$

Suppose that $\lambda_{m n} \neq 0$, that is,

$$
\begin{gathered}
\operatorname{Im} a \neq \frac{(-1)^{n}(n+1 / 2)}{2 m} \cdot \frac{l}{T}-\frac{2 m \pi}{l}, \\
n=0,1,2, \ldots, \quad m=0, \pm 1, \pm 2, \ldots \\
\text { If } n=2 k+1 \text { and } k=0,1,2, \ldots, \text { then } \\
\lambda_{m, 2 k+1}=-\left(2 k+\frac{3}{2}\right) \frac{\pi}{T}-\left(\frac{2 m \pi}{l}\right)^{2}-\frac{2 m \pi}{l} \operatorname{Im} a \longrightarrow+\infty
\end{gathered}
$$

at $m, k \rightarrow \infty$; therefore this subsequence has no limit points.

$$
\text { If } n=2 k, k=0,1,2, \ldots \text {, then }
$$

$$
\begin{array}{r}
\lambda_{m, 2 k}=\left(2 k+\frac{1}{2}\right) \frac{\pi}{T}-\left(\frac{2 m \pi}{l}\right)^{2}-\frac{2 m \pi}{l} \operatorname{Im} a, \\
k=0,1,2, \ldots, \quad m=0, \pm 1, \pm 2, \ldots .
\end{array}
$$

Transform this expression to a form convenient for us

$$
\begin{aligned}
\lambda_{m, 2 k} & =\frac{2 \pi}{T}\left[k+\frac{1}{4}-\frac{2 m^{2} \pi T}{l^{2}}-\frac{m T}{l} \operatorname{Im} a\right] \\
& =\frac{2 \pi}{T}\left[k+\frac{1}{4}-\left(m^{2} \frac{2 \pi T}{l^{2}}+m \frac{T \cdot \operatorname{Im} a}{l}\right)\right] .
\end{aligned}
$$

For convenience we introduce the following notation:

$$
\varphi(m)=m^{2} \frac{2 \pi T}{l^{2}}+m \frac{T \cdot \operatorname{Im} a}{l},
$$

where $[x]$ is the integer part and $(x)$ is the fractional part. Suppose that $k=[\varphi(m)]$, then

$$
\begin{aligned}
\lambda_{m, 2 k} & =\frac{2 \pi}{T}\left[\frac{1}{4}+[\varphi(m)]-\varphi(m)\right] \\
& =\frac{2 \pi}{T}\left[\frac{1}{4}-(\varphi(m)-[\varphi(m)])\right]=\frac{2 \pi}{T}\left[\frac{1}{4}-(\varphi(m))\right] .
\end{aligned}
$$

Now we use the Weyl's theorem [11], for this we assume that at least one of the values of

$$
\frac{2 \pi T}{l^{2}}, \quad \frac{T \cdot \operatorname{Im} a}{l}
$$

is irrational. Then by Weyl's theorem the fractional part $\varphi(m)$, that is, $(\varphi(m))$, fills the interval $[0,1]$ uniformly dense when $m=0,1,2, \ldots$. Then the subsequence $\left\{\lambda_{m, 2 k}\right\}, m=$ $0,1,2, \ldots, k=[\varphi(m)]$, is dense everywhere in the interval $[-3 \pi / 2 T, \pi / 2 T]$.

Assuming $k=[\varphi(m)]+1$ and $k=[\varphi(m)]+2, \ldots$, then $k=[\varphi(m)]-1, k=[\varphi(m)]-2, \ldots$, and so on; we obtain that the sequence $\left\{\lambda_{m n}\right\}$ is uniformly dense everywhere; that is, the continuous spectrum of the operator $\bar{L}$ fills the entire real axis from $-\infty$ to $+\infty$. Let now both values of

$$
\frac{2 \pi T}{l^{2}}, \quad \frac{T \cdot \operatorname{Im} a}{l}
$$

be rational; then $\varphi(m)$ is always rational. To be specific let

$$
\frac{2 \pi T}{l^{2}}=\frac{p}{q}, \quad \frac{T \cdot \operatorname{Im} a}{l}=\frac{r}{k} .
$$

Then

$$
\varphi(m)=m^{2} \cdot \frac{p}{q}+m \cdot \frac{r}{k}=\frac{m^{2} p+m r}{q \cdot k}=[\varphi(m)]+(\varphi(m)) .
$$

The fractional part $\varphi(m)$ takes only a finite number of values; they are the remainders of the division $m^{2} p+m r$ by $q \cdot k$; that is

$$
0, \frac{1}{q \cdot k}, \frac{2}{q \cdot k}, \ldots, \frac{q \cdot k-1}{q \cdot k} .
$$

When $m$ is changing from $-\infty$ to $+\infty$, these values will repeat infinitely many times, at least one or all of them. For us it is important that they do not coincide with $1 / 4$. 
Assuming $k=[\varphi(m)], m=0,1,2, \ldots$, we see that

$$
\lambda_{m, 2 k}=\frac{2 \pi}{T}\left[\frac{1}{4}-(\varphi(m))\right], \quad m=0,1,2, \ldots
$$

This infinite sequence is contained in a segment $[-3 \pi / 2 T, \pi / 2 T]$ and consists of a finite number of fractions of the form

$$
\begin{aligned}
& \frac{2 \pi}{T} \cdot \frac{1}{4}, \frac{2 \pi}{T} \cdot\left(\frac{1}{4}-\frac{1}{q \cdot k}\right), \frac{2 \pi}{T} \cdot\left(\frac{1}{4}-\frac{2}{q \cdot k}\right), \ldots, \\
& \frac{2 \pi}{T} \cdot\left(\frac{1}{4}-\frac{q k-1}{q \cdot k}\right),
\end{aligned}
$$

so at least one of them, or all, or some of them are repeated infinitely many times. This suggests that some numbers in the segment $[-3 \pi / 2 T, \pi / 2 T]$ are the infinitely multiple eigenvalues.

Continuing this reasoning as in the irrational case, we see that the spectrum of the operator $\bar{L}$ consists of an infinite set of eigenvalues and among the eigenvalues there are the infinite set of the infinitely multiple eigenvalues. By our assumption

$$
\begin{aligned}
& \operatorname{Im} a \neq \frac{(-1)^{n}(n+1 / 2)}{2 m} \cdot \frac{l}{T}-\frac{2 m \pi}{l}, \\
& n=0,1,2, \ldots, m=0, \pm 1, \pm 2, \ldots
\end{aligned}
$$

therefore the inverse operator exists and is bounded but is not compact in view of the existence of eigenvalues of infinite multiplicity, as the spectrum of the compact operator has a finite multiplicity. If $\operatorname{Re} a=0$ and

$$
\operatorname{Im} a=\frac{(-1)^{n}(n+1 / 2)}{2 m} \cdot \frac{l}{T}-\frac{2 m \pi}{l}
$$

for some values $n=0,1,2, \ldots, m=0, \pm 1, \pm 2, \ldots$, the inverse operator $\bar{L}^{-1}$ does not exist and zero is an eigenvalue, perhaps, of the infinite multiplicity. In this case, if at least one of the values of

$$
\frac{2 \pi T}{l^{2}}, \quad \frac{T \cdot \operatorname{Im} a}{l}
$$

is irrational, then the spectrum of the operator $\bar{L}$ fills the entire real axis. If both of these values are rational, then the spectrum of the operator $\bar{L}$ consists of an infinite number of eigenvalues, among which there are an infinite number of infinite multiple eigenvalues.

Finally, we note that the boundary value problem (2)-(4) is strongly solvable in the cases of (a) and (b) of Theorem 11 but in cases (c) and (d) is not.

\section{Conclusions}

By means of Fourier method, a criterion for strong solvability of the mixed Cauchy problem for the heat equation with a deviating argument was found. The nature of the spectrum of the periodic problem for the heat equation with a deviating argument was studied in detail, and the norm of the inverse operator was estimated through the influence coefficient. The dependence between the coefficient of influence and the nature of the spectrum of the heat operator with a deviating argument was found.

\section{Conflict of Interests}

The authors declare that there is no conflict of interests regarding the publication of this article.

\section{Acknowledgments}

The authors are grateful to the referees for their critical comments which helped in improving the content of this paper. The work is carried out under the auspices of the Ministry of Education and Science of Kazakhstan (1100/SF, 0754/SF).

\section{References}

[1] T. Sh. Kal'menov, S. T. Akhmetova, and A. Sh. Shaldanbaev, "On the spectral theory of equations with deviating argument," Matematicheskiu Zhurnal, vol. 4, no. 3, pp. 41-48, 2004 (Russian).

[2] M. A. Sadybekov and A. M. Sarsenbi, "Solution of fundamental spectral problems for all the boundary value problems for a first-order differential equation with a deviating argument," Uzbekskiu Matematicheskiu Zhurnal, no. 3, pp. 88-94, 2007 (Russian).

[3] A. A. Kopzhassarova, A. L. Lukashov, and A. M. Sarsenbi, "Spectral properties of non-self-adjoint perturbations for a spectral problem with involution," Abstract and Applied Analysis, vol. 2012, Article ID 590781, 5 pages, 2012.

[4] A. M. Samollenko and N. A. Perestyuk, Impulsive Differential Equations, vol. 14 of World Scientific Series on Nonlinear Science. Series A: Monographs and Treatises 14, World Scientific Publishing Co. Inc., River Edge, NJ, USA, 1995.

[5] H. Akça, J. Benbourenane, and V. Covachev, "Existence theorem for semilinear impulsive functional differential equations with nonlocal conditions," International Journal of Applied Physics and Mathematics, vol. 3, no. 3, pp. 182-187, 2013.

[6] A. Kopzhassarova and A. Sarsenbi, "Basis properties of eigenfunctions of second-order differential operators with involution," Abstract and Applied Analysis, vol. 2012, Article ID 576843, 6 pages, 2012.

[7] T. Li, Z. Han, C. Zhang, and H. Li, "Oscillation criteria for second-order superlinear neutral differential equations," Abstract and Applied Analysis, vol. 2011, Article ID 367541, 17 pages, 2011.

[8] J. Wu, Theory and Applications of Partial Functional-Differential Equations, vol. 119 of Applied Mathematical Sciences, Springer, New York, NY, USA, 1996.

[9] M. Reed and B. Simon, Methods of Modern Mathematical Physics-vol. 1-Functional Analysis, Academic Press, London, UK, 1972.

[10] W. Rudin, Functional Analysis, McGraw-Hill, New York, NY, USA, 2nd edition, 1991. 
[11] H. Weyl, "Über die Gleichverteilung von Zahlen mod. Eins," Mathematische Annalen, vol. 77, no. 3, pp. 313-352, 1916.

[12] G. Y. Shilov, Mathematical Analysis. A Special Course, Pergamon Press, Oxford, UK, 1965. 


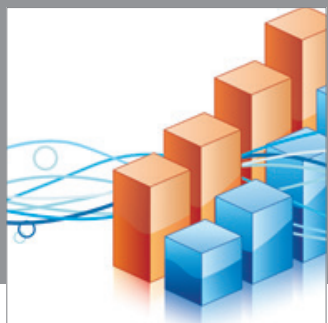

Advances in

Operations Research

mansans

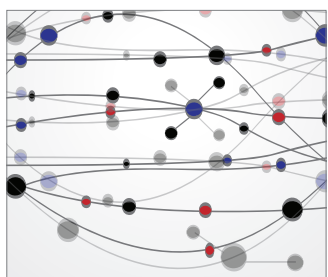

The Scientific World Journal
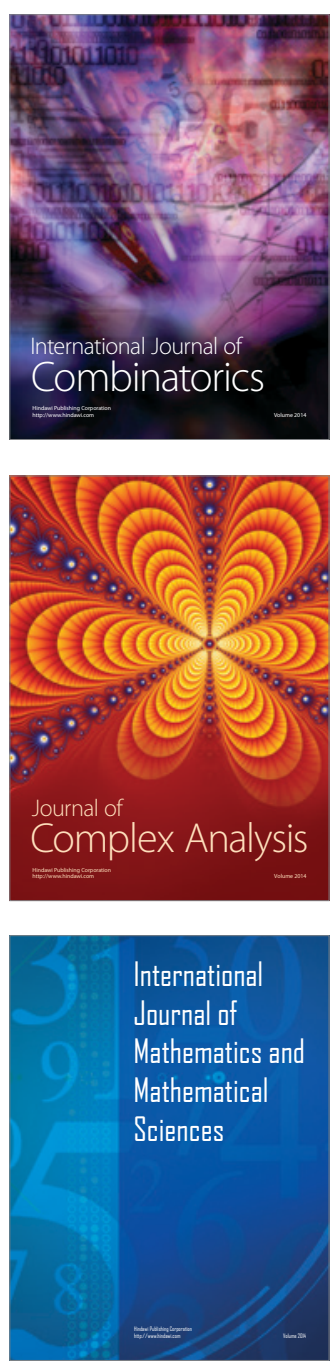
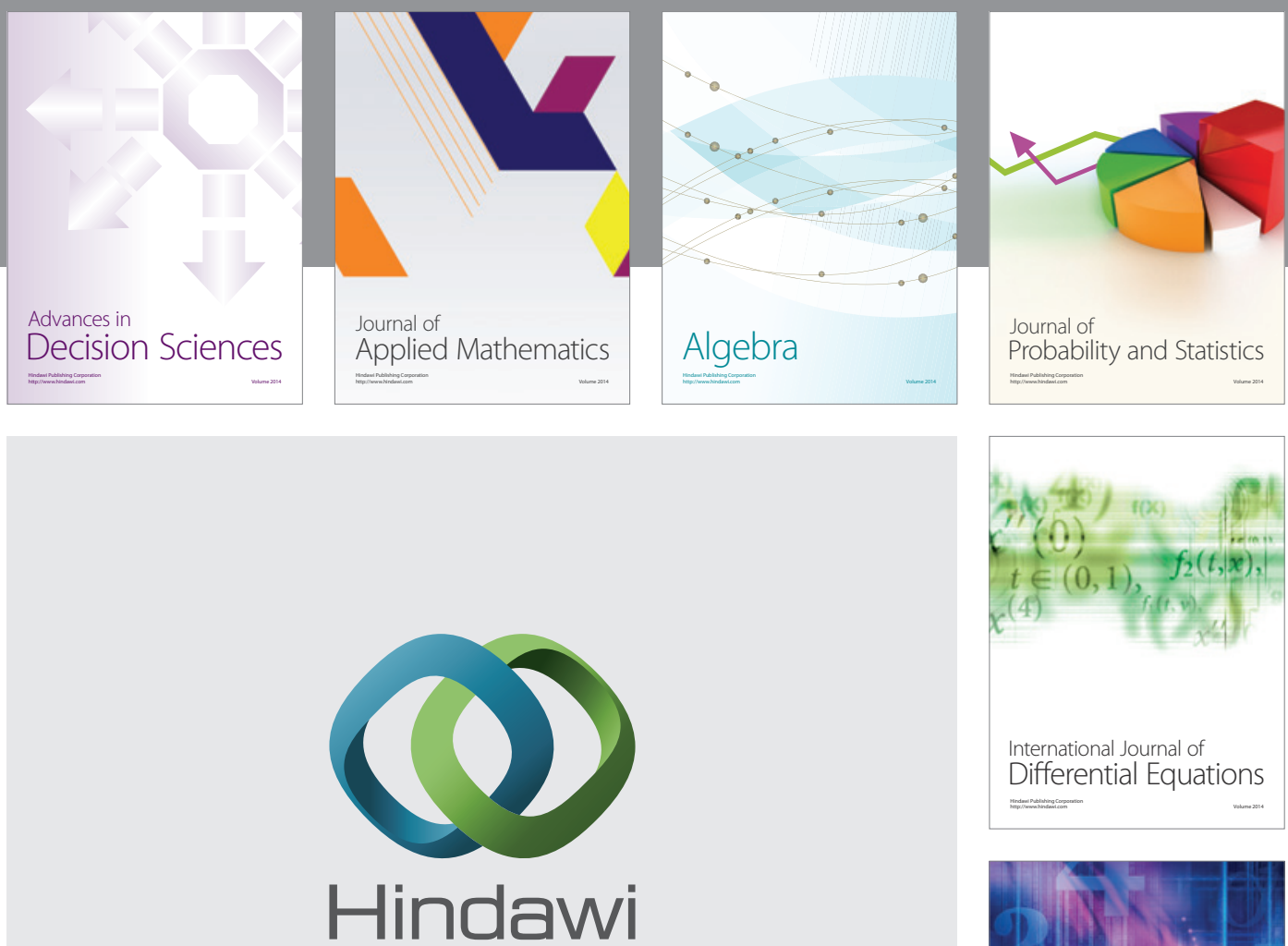

Submit your manuscripts at http://www.hindawi.com
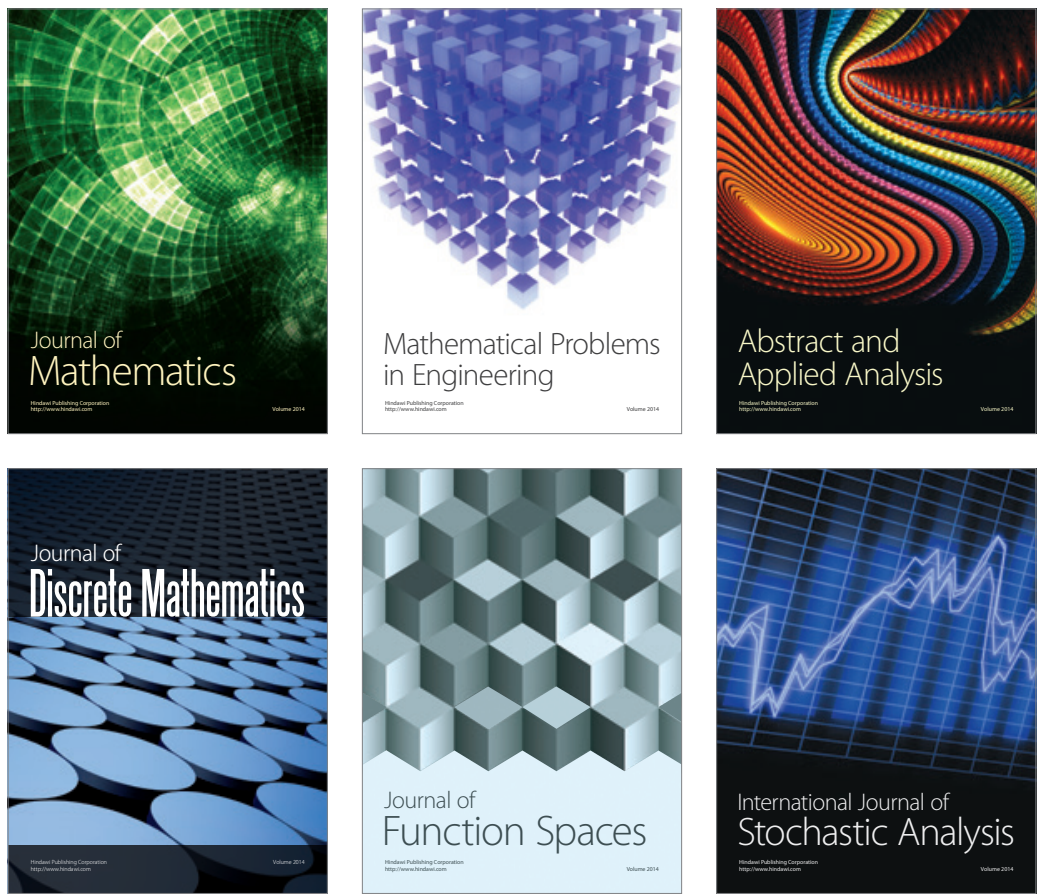

Journal of

Function Spaces

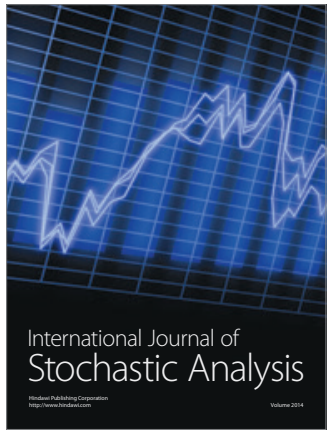

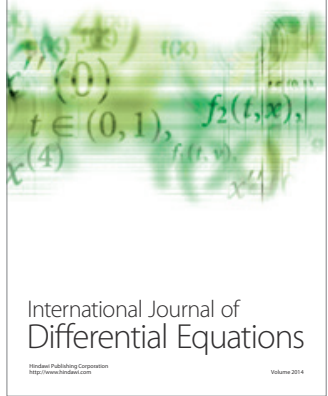
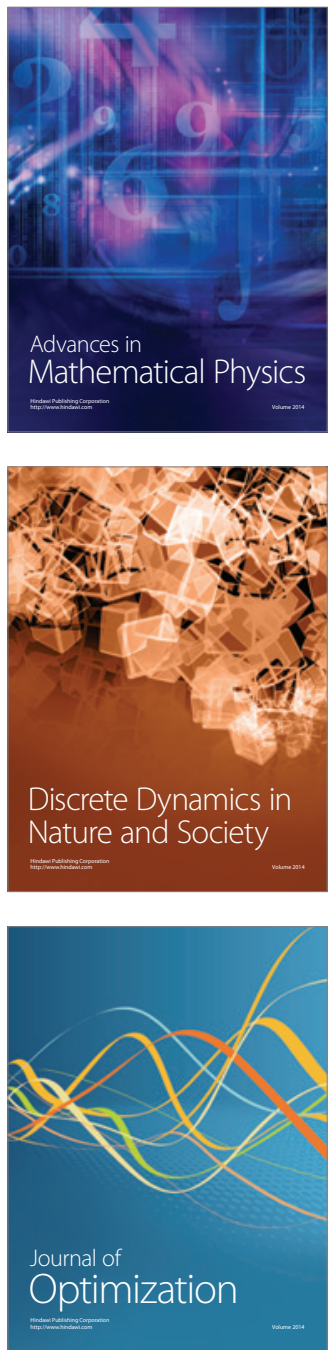